in the bright bands appearing at a later stage is unknown as yet, as also the connection between these maxima and the condensations or nuclei observed as the nova grows into a measurable disk or breaks into several nuclei:

A further line of study well worth following out at this stage would be an intercomparison of the various stages of development of different novae, such as the one that Dr McLaughlin has recently published on their rates of development. This is becoming increasingly possible with the wider publication of the observations of earlier novae.

The ultimate fate of novae and their links, if any, with white dwarfs and planetary nebulae have yet to be established. Differences between novae and super-novae need to be explored; they are almost certain to exist in other matters than the scale of the outburst. It cannot be said that any link between novae (or even supernovae) with cosmic rays has yet been established, and the committee feel that the subject of cosmic rays-perhaps now ripe for study by the methods of astronomyshould be placed under the care of some other committee.

F. J. M. Stratton

Chairman

\title{
REPORT OF SUB-COMMITTEE ON THE CLASSIFICATION OF WOLF-RAYET STARS
}

The Sub-Committee repeats its recommendations of r935 (see pp. I84-87 of Transactions of the International Astronomical Union, 5, I935). Mrs Gaposchkin, who at that time submitted a minority report, now expresses the opinion that: "It will be well to adopt the classification that he [Dr Beals] has proposed"; so that this recommendation may now be regarded as unanimous.

The following report has been submitted by Dr C. S. Beals:

\section{INTRODUCTION}

At the I935 meeting of the International Astronomical Union a tentative report was presented which dealt with the general problem of the classification of WolfRayet stars. The division of Wolf-Rayet stars into parallel sequences was demonstrated and criteria of classification were suggested which made it possible to include all Wolf-Rayet stars whose spectra had been adequately investigated. At that time it was not found possible to secure unanimous agreement in all points relating to the classification, and it was deemed best to postpone official action until certain additional observations could be made and until the question of nomenclature and notation could receive further consideration.

Since that time the observational basis of the classification has been strengthened, particularly in the visible region, where some of the most important criteria of classification are to be found. The accompanying illustration shows that the gap in the series of spectra accompanying the 1935 report has been filled by a grating spectrogram of the star $\mathrm{HD}_{\mathbf{5}} \mathrm{I}_{932}$ in the visible region. The new observations have amply confirmed the soundness of the original criteria of classification and there now seems no reason why the adoption of this system of classification should be further postponed. The report which follows is closely similar to that presented in I935. The principal difference concerns the matter of notation, where a more detailed discussion is presented and where a somewhat different approach to the question is advocated. 
WOLF-RAYET STARS, NITROGEN SEQUENCE

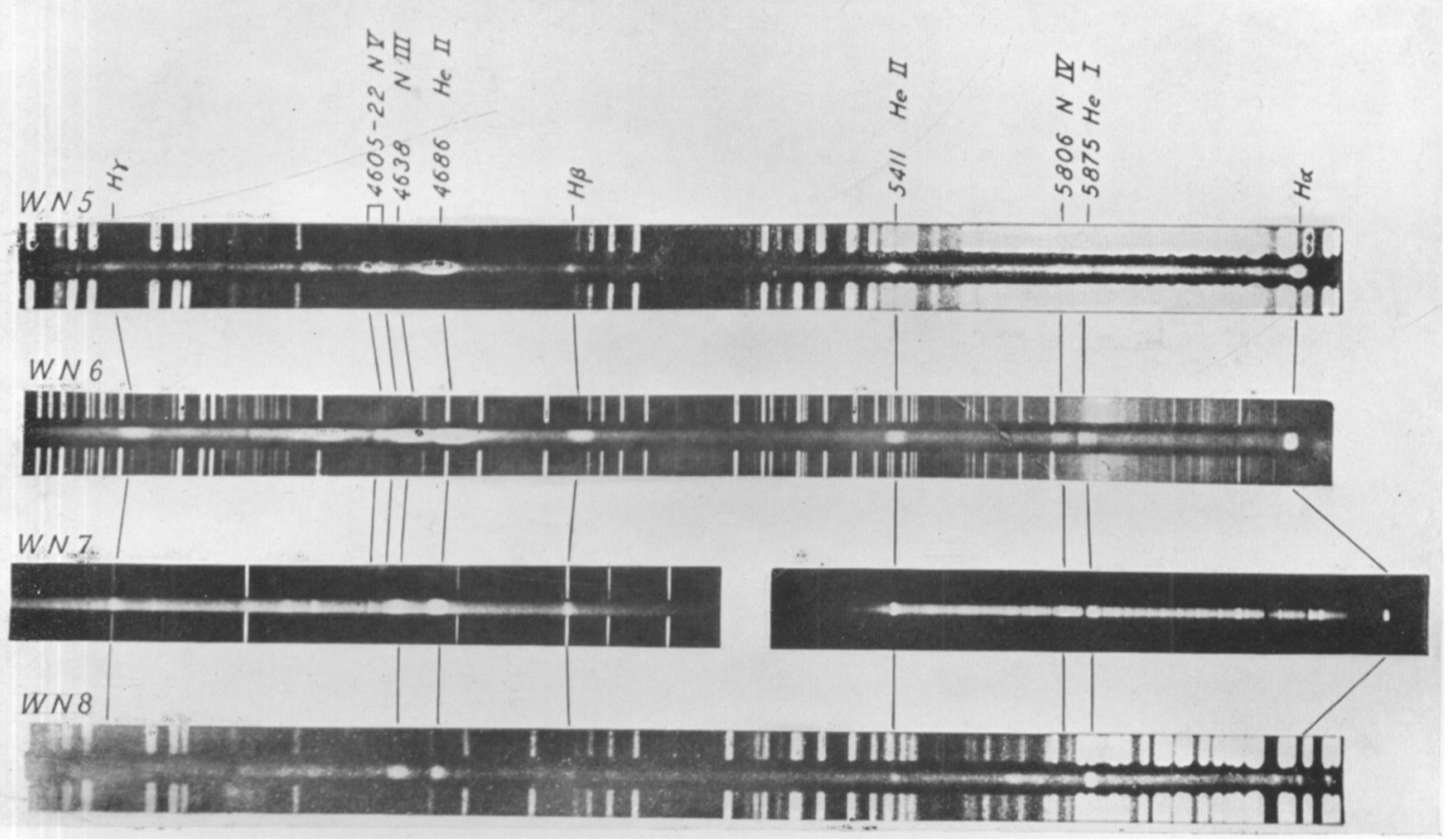

WN 5, HD I87282; WN 6, HD $192163 ; W_{7}, \mathrm{HD}_{151932 ;} \mathrm{WN}_{8}, \mathrm{HD}_{177230}$

WOLF-RAYET STARS, CARBON SEQUENCE

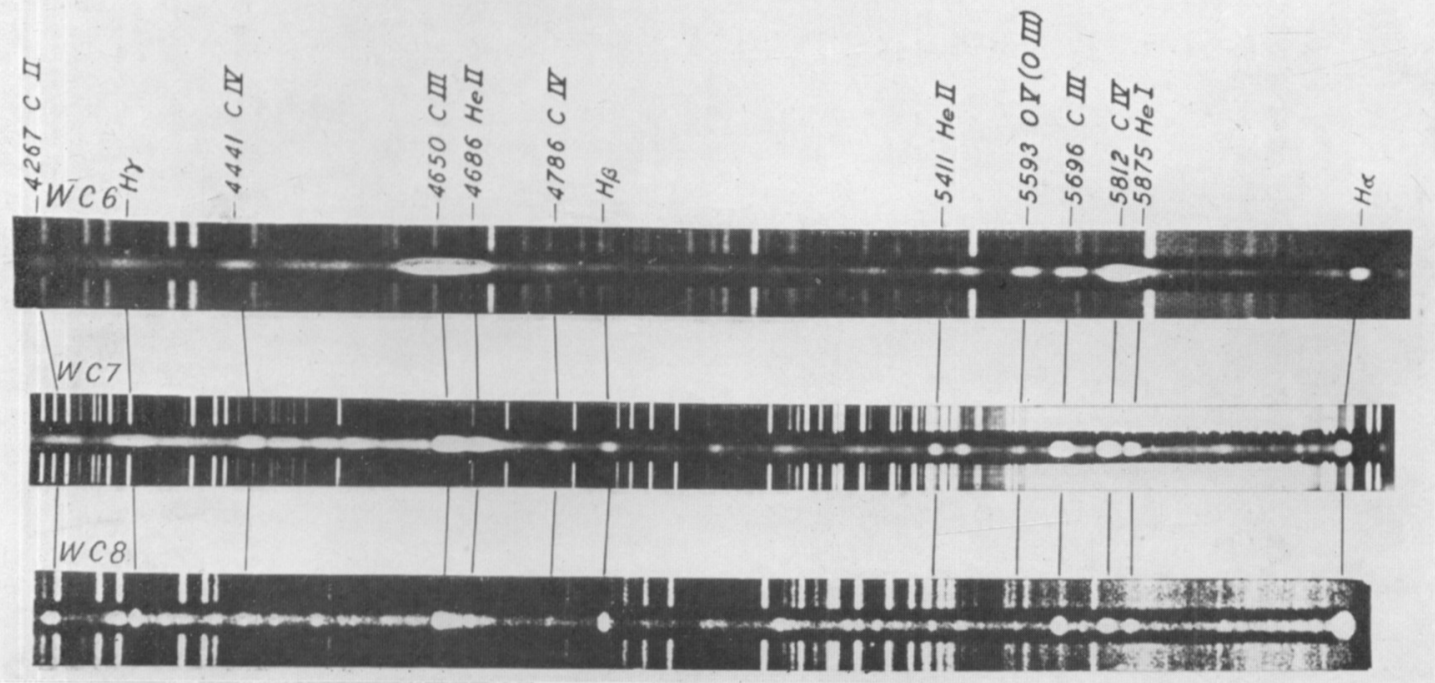

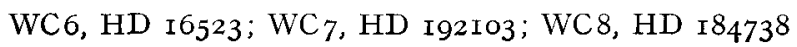




\section{Tentative Definition}

The Wolf-Rayet stars constitute an exceedingly interesting class of emissionline objects, the first three examples of which (HD I9I765, HD r92ro3 and HD I9264I) were discovered by Wolf and Rayet* at the Paris Observatory in I867. The spectrum of a typical Wolf-Rayet star, as exemplified by the three objects mentioned above, consists of a continuous spectrum on which are superposed numerous strong emission bands due to atoms of high ionization potential, the most important in the ordinary region being identified with the atoms HeI, HeII, CII, CIII, CIV, NIII, NIV and NV. The relationships existing between different members of the class are rather complicated, and it is impossible to give a completely unambiguous definition of a Wolf-Rayet star apart from a detailed description of the spectrum. Perhaps the best that can be done in a short space is to say that a Wolf-Rayet star is one that finds a logical place in one or the other of two clearly developed sequences of stars, of which $\mathrm{HD}$ I9I765 and HD I92Io3 are typical examples.

\section{The Two Parallel Sequences}

Since it has been demonstrated that the Wolf-Rayet stars may be divided into two parallel sequences of approximately the same general level of ionization, $\dagger$ a discussion of classification will probably be more intelligible if the two sequences are first separately described. For the sake of brevity the two sequences will be designated as the carbon sequence and the nitrogen sequence, respectively. The general characteristics of the two sequences may briefly be described as follows.

The Carbon Sequence. In addition to $\mathrm{HeI}$ and HeII which are common to the spectra of both sequences, the carbon sequence has as the most important constituents of its spectrum bands due to CII, CIII and CIV, OII, OIII, OIV, OV and OVI. Silicon is also present in various stages of ionization, as are also CaII, MgII and, probably, SII, but they occupy a relatively unimportant position as compared with oxygen and carbon. The wave-lengths in angstroms of principal bands representing the successive spectra of oxygen and carbon are as follows: CII, 4267; CIII, 3609, 4I87, 4325, 4650 and 5696; CIV, 444I, 4658 (blended with $4650), 4786$ and 58I2 ; OII, 4I54, 43I7, 4349, 4366, 44I4 and 44I7; OIII, 37I4, 3760, 396r; OIV, 3385, 3405, 34I2, 3562, 3725, 3736; OV, 5470, 5592; OVI, 38I5, 3835 .

In the ordinary photographic region the most striking feature of spectra of the carbon sequence is the appearance of very strong bands due to CIII at $\lambda_{4650}$ and HeII at $\lambda 4686$. In the visible regions strong bands due to CIII at $\lambda 5696$ and to CIV at $\lambda_{5812}$, accompanied by weaker ones at 54II, 5470, 5593 and 5875 , are characteristic features of the spectrum. There appears to be a definite and clearly marked relation between band width and spectral type in stars of the sequence. The band widths vary from approximately $80 \mathrm{~A}$. in stars of earliest type to some Io A. or less for those of lowest excitation. There is no clear indication of nitrogen in any of the stars of the carbon sequence.

The Nitrogen Sequence. Apart from bands due to $\mathrm{HeI}$ and HeII which are common to both sequences, the important feature of the nitrogen sequence is the presence of bands due to NIII, NIV and NV. The wave-lengths in angstroms of the most important nitrogen bands are as follows: NIII, 4099,§ 4640; NIV, 3483 , $4057,4939,5806 ; \mathrm{NV}, 4605,4622,4945$.

* Comptes Rendus, 65, 292, I867.

$\dagger$ ¿s.f. Astrophysik, 7, 1, 1933; Pub. D.A.O. 6, тзо, 1932.

† Blended band.

$\S$ Blended with $\mathrm{H} \delta$. 
In the ordinary region the most conspicuous feature of the spectra of members of this sequence is the appearance of 4686 of HeII in company with various bands due to nitrogen. Members of the Pickering series are present but are of less intensity than 4686 . In the lowest excitation stars the NIII band at 4640 is conspicuous and is of intensity comparable to 4686 . Passing along the sequence to earlier types 4640 grows weaker and is replaced by a broad composite band stretching from approximately 4600 to 4660 . It is probable that NIII (4640) and NV (4605-4622) contribute to the intensity of this band, but they are not clearly distinguishable as separate wave-lengths. In the earliest types of all the broad band disappears and its place is taken by two conspicuous narrow bands due to NV at 4605-4622. These bands are usually accompanied by a weaker band at 4945 due to the same atom.

In the visible region bands at $54 \mathrm{II}$ due to $\mathrm{HeII}$, at $5^{875}$ due to $\mathrm{HeI}$, and a band at 5806 due to NIV, are the important features. The bands $54 \mathrm{II} \mathrm{HeII}$ and $5875 \mathrm{HeI}$ are unblended and so form convenient criteria of excitation.

\section{NOMENCLATURE}

In the first Draper Catalogue* the single Wolf-Rayet star included was designated as Class $\mathrm{O}$, and it was at that time apparently the only star so designated. Later, in the H.D. Catalogue, the letter $\mathrm{O}$ was used for both emission and absorption stars of early type. Since the publication of the H.D. Catalogue a new decimal system has been adopted for the designation of absorption line stars of the earliest spectral type, now generally referred to as Class $0 . \dagger$ While the Wolf-Rayet stars and the absorption $\mathrm{O}$ stars are probably of much the same general level of ionization, there are important differences between them which are not obviously related to the bright or dark line character of their spectra. Accordingly, in connection with other proposals for a scheme of classification for these objects, it has seemed desirable to put forward a new symbol or set of symbols to designate the WolfRayet class. If the Wolf-Rayet stars were a homogeneous group of objects as regards the chemical elements appearing in their spectra, this problem could be solved by employing a single letter, such as $\mathrm{W}$, to designate the class. The existence of the carbon and nitrogen sequences introduces obvious complications in nomenclature and two different suggestions have been advanced as possible solutions of the difficulty. These suggestions are separately discussed as follows:

(I) The carbon and nitrogen sequences could be distinguished by separate letters such as $U$ and $V$ or $W$ and $X$. The principal objection to this suggestion lies in the possibility of confusion arising because of the existence of a third parallel sequence in the absorption O's. If this suggestion were adopted there would then be three symbols of a uniform character, $\mathrm{O}, \mathrm{U}, \mathrm{V}$, designating early type stars without a uniform basis of distinction between them (i.e. the distinction between $\mathrm{O}$ and $\mathrm{V}$ would be mainly concerned with emission and absorption, while the distinction between $\mathrm{U}$ and $\mathrm{V}$ would be concerned with chemical constitution).

(2) Under these circumstances it seems best to employ the letter W (which suggests the discoverers and does not interfere with any previously established usage) to designate the Wolf-Rayet class and to use subsidiary symbols of some sort to distinguish between the carbon and nitrogen sequences. The problem then reduces to finding suitable symbols for this purpose.

$$
\text { * H.A.27. }
$$


In the I935 report the use of the symbols $W$ and $W^{\prime}$ (prime) was suggested for the nitrogen and carbon sequences respectively. Since then, this suggestion has been criticized as being too arbitrary and not sufficiently descriptive. It is very easy, for example, to confuse the two and forget which is $W$ and which $W^{\prime}$. This criticism can be met by making use of the chemical symbols for carbon and nitrogen in the form of small capitals or italicized capitals $C$ and $N$. The lower case letters $\mathrm{c}$ and $\mathrm{n}$ are already in use in the Draper classification system, but they apply only to absorption line spectra and it seems possible by altering the character of the letters and the positions in which they are placed to avoid all possibility of confusion. When the letter $c$ is used to designate an absorption line spectrum of a particular type the lower case is employed and the letter is placed before the main classification symbol, i.e. cA3. When the letter $\mathrm{n}$ is similarly used to indicate absorption line character it follows the subdivision number of the stars, i.e. B2n. The chemical symbols $\mathrm{N}$ and $\mathrm{C}$ can be made sufficiently distinctive, first by using small superior capitals or italicized capitals instead of lower case letters and, secondly, by so placing them that they can hardly be confused with other similar symbols now in use. Suggestions which have been made are the use of subscripts $\left(\mathrm{W}_{\mathrm{N}} 8, \mathrm{~W}_{\mathrm{C}} 8\right)$, superscripts $\left(\mathrm{W}^{\mathrm{N}} 8, \mathrm{~W}^{\mathrm{C}} 8\right.$ ), or simply placing the chemical symbol on the same line with the main symbol before the subdivision number of the star (WN8, Wc8). For ease in the preparation of manuscript and from a typographical standpoint the third suggestion probably has most to recommend it. It is probably best to regard the use of the chemical symbols as to some extent optional. In statistical discussions instances may arise where it may be considered desirable to dispense with them and use only the letter $W$ with the appropriate subdivision numbers of the stars. It is also possible that instances may be discovered where carbon and nitrogen appear in the same spectrum, and under these circumstances the letter W without the chemical symbols could be used.

In the great majority of cases, however, it is only possible to gain a clear idea of the character of a Wolf-Rayet spectrum when both the sequence to which it belongs and its subdivision number are known. It is accordingly strongly recommended that the chemical symbols be employed in the manner outlined above whenever it is at all feasible to do so. In this way this important cleavage which exists among Wolf-Rayet stars will be clearly illustrated and the nature of the symbols used should make it possible, without undue strain on the memory, to make it quite certain to which sequence a particular star belongs.

Grouping of Stars in Subdivisions: The Nitrogen Sequence. For the purpose of subdividing the nitrogen sequence there are available the following criteria of excitation:

(I) NIII/HeII ratio: available over lower range of sequence; bands employed 4640 NIII and 4686 HeII.

(2) Presence of broad band between 4600 and 4660 . This must be regarded as a subsidiary criterion of excitation because the band is not certainly identified. It is clear and unambiguous, however, and enables many Wolf-Rayet stars to be classified at a glance. Useful in one subdivision only.

(3) NV/HeII ratio: bands employed $4605-4622$ of NV and 4686 of HeII. Useful in one subdivision only. Upper part of sequence.

(4) $\mathrm{HeI} / \mathrm{HeII}$ ratio: available throughout the sequence. Bands employed $5875 \mathrm{HeI}$ and 54II HeII.

The spectra of the nitrogen sequence have been grouped into four subdivisions which, in accordance with the discussion of nomenclature, have been designated as 
WN5, WN6, WN7 and WN8. The numbering has been so chosen as to allow a place for new discoveries at either end of the sequence. The values given for intensity ratios are subject to a degree of latitude depending upon the amount of variation from class to class. A list of the subdivisions with excitation criteria and typical stars follows.

Class WN5: Ratios $4605-4622 / 4686=0 \cdot 2,5875 / 54 I I=0 \cdot I ; 4945$ present. Typical stars HD 187282 and HD 2 II 56.

Class WN6: Ratios $5875 / 54 \mathrm{II}=0.5$; band $4600-4660$ present and strong; 4938 present. Typical stars HD I9I765 and HD I92I63.

Class WN7: Ratios $4640 / 4686=0 \cdot 5,5875 / 54 \mathrm{II}=\mathrm{I} \cdot 5$. Typical stars HD I5I932 and $\mathrm{HD} 92740$.

Class WN8: Ratios $4640 / 4686=I \cdot 5 ; 5^{875} / 54 I I=5^{\circ}$. Typical stars HD I77230 and HD 96548 .

Grouping of Stars in Subdivisions: The Carbon Sequence. For the purpose of subdividing the carbon sequence there are available the following criteria of excitation:

(I) CII/CIV ratio; available only over the lower range of sequence; bands employed $4267 \mathrm{CII}$ and $4786 \mathrm{CIV}$.

(2) CIII/CIV ratio; available throughout the sequence; bands employed 5695 CIII and $58 \mathrm{I} 2 \mathrm{CIV}$.

(3) CIII/OV ratio; useful only in the upper range of the sequence; bands employed $5696 \mathrm{CIII}$ and $5592 \mathrm{OV}$.

(4) CIII/HeII ratio; available in the lower range of the sequence; bands employed $4650 \mathrm{CIII}$ and $4686 \mathrm{HeII}$.

(5) HeI/HeII ratio; available in the lower range of the sequence; bands employed $5875 \mathrm{HeI}$ and $54 \mathrm{II} \mathrm{HeII}$.

(6) Band width. The correlation between band width and spectral type in the carbon sequence has already been mentioned. This correlation is so clearly marked and consistent that estimates of band width form a useful subsidiary criterion of excitation. This is especially true of the earlier types, where some of the bands ordinarily resolved are blended to form a single band.

The spectra of the carbon sequence have been grouped into three subdivisions which, in accordance with the discussion on nomenclature, have been designated respectively Wc6, Wc7, Wc8. The numbering has been chosen so as to allow a certain latitude for new discoveries at either end of the sequence. The values given for intensity ratios must be regarded as approximate means for the class, and subject to a degree of latitude which depends on the value of the ratio in the preceding and following groups. The band widths given are approximate. The edge of the band is taken as the point where its intensity drops to $0 \cdot I$ of its central value.

The newly suggested groups with intensity ratios or other excitation criteria and standard stars representing each class are as follows:

Class Wc6: Ratios $5696 / 5812=0 \cdot 3,5696 / 5592=1 \cdot 2,4267 / 4786=0 \cdot 0,4650$ and 4686 not resolved, 5812 and 5875 not resolved. Band width approximately $70 \mathrm{~A}$. Typical stars HD 16523 and $\mathrm{HD}$ x65763.

Class Wc7: Ratios 5696/58I $2=0 \cdot 7,5696 / 5592=8 \cdot 0,5875 / 54 \mathrm{II}=\mathrm{I} \cdot 5,4650 / 4686=$ $4 \cdot 0,4267 / 4786=\mathrm{I} \cdot 0,4650$ and 4686 just resolved. Band width approximately 35 A. Typical stars HD 192103 and HD 199078.

Class Wc 8 : Ratios 5696/58I2 $=3^{\circ} 0,5875 / 54 \mathrm{II}=5^{\circ} 0,4650 / 4686=9 \cdot 0,4267 / 4786=$ 2.0. Band width approximately Io A. Typical stars HD I84738 and HD I64270. 
It may be noted that in the two lower sub-classes $W_{C 7}, W_{C} 8$ and $W_{N} 7, W_{N} 8$ the classification intensity ratio $5875 / 54$ II has the same value for both carbon and nitrogen sequences, indicating that they are of closely comparable excitation. In the carbon sequence the ratio $5875 / 54$ II cannot be used above subdivision $\mathrm{WC} 7$, owing to blending of $\lambda_{5875}$ with the strong band at $\lambda_{5812}$.

C. S. Beals

Chairman

The reports of these sub-committees and the suggestions of Drs Merrill and Struve appear to be the only matters upon which this Commission should vote when in session at Stockholm.

HENRY NORRIS RUSSELL

President of the Commission 